\title{
Risk of Covid-19-Related Hospitalization and More Severe Outcomes in Medicare Beneficiaries Treated with Renin-Angiotensin-Aldosterone System Inhibitors for Hypertension
}

\author{
David J. Graham, MD, MPH ${ }^{1}$, Hector S. Izurieta, MD, PhD ${ }^{2}$, Stella G. Muthuri, PhD, MPH ${ }^{3}$, \\ Di Zhang, PhD', Alexander T. Sandhu, MD, MS 3,5 , Yun Lu, PhD', Yueqin Zhao, PhD4, \\ Yuhui Feng, $M S^{3}$, Efe Eworuke, $P h D^{7}$, Hai $L y u, M S^{3}$, Charu Gandotra, $M D^{7}$, \\ Elizabeth R. Smith, BS ${ }^{3}$, Armen Avagyan, $\mathrm{MS}^{3}$, Michael Wernecke, BA ${ }^{3}$, \\ Jeffrey A. Kelman, MD, MSSc ${ }^{8}$, Richard A. Forshee, PhD', and Thomas E. MaCurdy, \\ $P h D^{3,9}$
}

\begin{abstract}
'Office of Surveillance and Epidemiology, Center for Drug Evaluation and Research, US Food and Drug Administration, 10903 New Hampshire Ave, Building 22, Room 4314, Silver Spring, MD, USA; ${ }^{2}$ Office of Vaccines Research and Review, Center for Biologics Evaluation and Research, US Food and Drug Administration, Silver Spring, MD, USA; ${ }^{3}$ Acumen LLC, Burlingame, CA, USA; ${ }^{4}$ Office of Biostatistics, Center for Drug Evaluation and Research, US Food and Drug Administration, Silver Spring, MD, USA; ${ }^{5}$ Division of Cardiology, Department of Medicine, Stanford University, Stanford, CA, USA; ${ }^{6}$ Office of Biostatistics and Epidemiology, Center for Biologics Evaluation and Research, US Food and Drug Administration, Silver Spring, MD, USA; ${ }^{7}$ Office of New Drugs, Center for Drug Evaluation and Research, US Food and Drug Administration, Silver Spring, MD, USA; ${ }^{8}$ Centers for Medicare \& Medicaid Services, Washington, DC, USA; ${ }^{9}$ Department of Economics, Stanford University, Stanford, CA, USA.
\end{abstract}

BACKGROUND: There are theoretical concerns that angiotensin-converting enzyme inhibitors (ACEIs) and angiotensin receptor blockers (ARBs) could increase the risk of severe Covid-19.

OBJECTIVE: To determine if ACEIs and ARBs are associated with an increased risk of Covid-19 hospitalization overall, or hospitalization involving intensive care unit (ICU) admission, invasive mechanical ventilation, or death.

DESIGN: Observational case-control study.

PARTICIPANTS: Medicare beneficiaries aged $\geq 66$ years with hypertension, treated with ACEIs, ARBs, calcium channel blockers (CCBs), or thiazide diuretics.

MAIN MEASURES: Adjusted odds ratios (OR) and 95\% confidence intervals (CI) for the outcomes of Covid-19 hospitalization, or hospitalization involving ICU admission, invasive mechanical ventilation, or death.

RESULTS: A total of 35,300 cases of hospitalized Covid19 were matched to 228,228 controls on calendar date and neighborhood of residence. The median age of cases was 79 years, $57.4 \%$ were female, and the median duration of hospitalization was 8 days (interquartile range 512). ACEIs and ARBs were associated with a slight reduction in Covid-19 hospitalization risk compared with treatment with other first-line antihypertensives (OR for ACEIs 0.95, 95\% CI 0.92-0.98; OR for ARBs 0.94, 95\% CI 0.900.97). Similar results were obtained for hospitalizations involving ICU admission, invasive mechanical ventilation, or death. There were no meaningful differences in risk for ACEIs compared with ARBs. In an analysis restricted to monotherapy with a first-line agent, CCBs were associated with a small increased risk of Covid-19 hospitalization

Received April 12, 2021

Accepted September 14, 2021

Published online October 1, 2021 compared with ACEIs (OR 1.09, 95\% CI 1.04-1.14), ARBs (OR 1.10, 95\% CI 1.05-1.15), or thiazide diuretics (OR $1.11,95 \%$ CI 1.03-1.19).

CONCLUSIONS: ACEIs and ARBs were not associated with an increased risk of Covid-19 hospitalization or with hospitalization involving ICU admission, invasive mechanical ventilation, or death. The finding of a small increased risk of Covid-19 hospitalization with CCBs was unexpected and could be due to residual confounding.

KEY WORDS: angiotensin-converting enzyme inhibitors; angiotensin receptor blockers; Covid-19; intensive care; mechanical ventilation.

J Gen Intern Med 36(12):3802-9

DOI: $10.1007 /$ s11606-021-07155-Z

(C) This is a U.S. government work and not under copyright protection in the U.S.; foreign copyright protection may apply 2021

\section{INTRODUCTION}

Coronavirus disease 2019 (Covid-19) is caused by severe acute respiratory syndrome coronavirus 2 (SARS-CoV-2), which establishes infection by binding of its envelope spike protein to the angiotensin-converting enzyme 2 (ACE2) receptor on the surface of respiratory epithelium. ${ }^{1}$ In humans, ACE2 converts angiotensin II, a proinflammatory vasoconstrictor, into angiotensin-(1-7), a vasodilator with antiinflammatory properties. ${ }^{2,3}$ In some animal models, treatment with angiotensin-converting enzyme inhibitors (ACEIs) and angiotensin receptor blockers (ARBs) upregulates the expression of ACE2, leading to concern that such upregulation, if it occurred in humans, might increase susceptibility to SARSCoV-2 infection in patients treated with these medications. ${ }^{2}$ 
Also, binding of SARS-CoV-2 to the ACE2 receptor leads to downregulation of ACE2 receptor density, which may contribute to angiotensin II accumulation and increased pulmonary toxicity. ${ }^{4}$ Given that over $20 \%$ of the US population aged 60-79 years are treated with ACEIs, ${ }^{5}$ the public health importance of understanding whether ACEIs and ARBs alter Covid19 risk or severity is great.

Since the beginning of the pandemic, many observational studies of varying quality have explored the association of ACEIs and ARBs on the risk of developing severe Covid-19. Mackey and colleagues performed a living systematic review of published observational studies on this topic, with monthly updates. ${ }^{6}$ While the conclusion of this review was that ACEIs and ARBs are not associated with an increased risk of developing Covid-19 or of experiencing a more severe outcome, most of the studies cited were relatively small, limited to single institutions or geographic regions, and conducted in countries other than the USA. ${ }^{6}$ Only a handful of larger, population-based studies of Covid-19 risk in association with ACEI or ARB use have been reported. ${ }^{7-17}$ Despite their population-based nature, many were too small to exclude clinically important risks and none examined individually the full range of outcome severity, including intensive care unit (ICU) admission, invasive mechanical ventilation use, and Covid-related death. Only 4 of these studies were conducted in the USA, ${ }^{7,8,13,16}$ of which 3 were limited to a single closed healthcare system and employed nonusers as the reference, ${ }^{7,8,13}$ thereby increasing the possibility of confounding by indication ${ }^{18}$ as well as confounding by hypertension severity and related comorbidities. The other US study involved a single national commercial insurance plan and compared patients treated with ACEIs and ARBs with patients treated with any other antihypertensive agent. ${ }^{16}$ The US studies were relatively small in size, did not examine ACEI and ARB risk in subgroups defined by race or nursing home residence, and did not examine risk across the full range of outcome severity.

To address the limitations of previous studies, we performed a nationally representative case-control study of the risk of Covid-19 hospitalization or hospitalization involving ICU admission, invasive mechanical ventilation, or death, within the population of older patients enrolled in fee-for-service Medicare, diagnosed with hypertension, and treated with ACEIs, ARBs, or another first-line antihypertensive medication.

\section{METHODS}

Medicare provides health insurance coverage to persons aged 65 years and older as well as to persons under age 65 who have end-stage kidney disease or are disabled. We performed a case-control study in the population of Medicare beneficiaries who were 66 years of age or older; had at least 1 year of enrollment in fee-for-service Medicare Part A (hospitalization), Part B (office-based care), and Part D (prescription drug coverage); had a diagnosis of hypertension; and had a recently filled prescription for a first-line antihypertensive agent
(ACEIs, ARBs, calcium channel blockers (CCBs), thiazide diuretics). In March 2020, this population included 9.8 million patients.

The study period ran from April 1, 2020 (the date when the International Classification of Diseases, Tenth Revision, Clinical Modification (ICD-10-CM) code specific for Covid-19 (U07.1) became effective), through July 28, 2020. Over this period, we identified all patients in the study population with an incident ICD-10-CM inpatient hospital discharge diagnosis of Covid-19 in any diagnosis position. Patients with unconfirmed probable or suspected Covid-19 hospitalizations prior to study start were excluded. The date of Covid-19 hospital admission was defined as the index date for matching with controls (Supplement Figure 1).

Incidence density sampling with replacement was used to randomly select up to 10 controls per case,${ }^{19}$ matched on index date and neighborhood of residence (determined by census block or block group), from the base population restricted to those who had not been previously hospitalized for Covid-19. Given that the intensity of the Covid-19 pandemic across the USA varied temporally and geospatially, this matching strategy ensured that cases and controls had similar opportunity of being exposed to SARS-CoV-2.

Over the 365 days preceding the index date, data for each case and control were collected on demographics, medical comorbidities, chronic medications, and healthcare utilization (Table 1, Supplement Table 1). We also estimated the frailty score (a validated measure of patient frailty), ${ }^{20,21}$ the Charlson comorbidity index (a validated metric that correlates with the probability of death in the subsequent year), ${ }^{22,23}$ and the adapted diabetes complications severity index (aDCSI; a validated measure of diabetes severity). ${ }^{24,25}$

Treatment with a first-line antihypertensive drug was defined as having a recently filled prescription that provided $\geq 15$ days of medication supply during the 30 days prior to the index date. The exposure of interest was treatment with an ACEI (without $\mathrm{ARB}$ ) or ARB (without ACEI), and these patients could also be receiving CCBs and/or thiazide diuretics. The comparator exposure was defined as treatment with a $\mathrm{CCB}$ and/or thiazide diuretic, without treatment with an ACEI or ARB. All patients could also be treated with non-first-line antihypertensives. A study from early in the pandemic suggested that CCBs and thiazides were neutral with respect to Covid-19 risk. ${ }^{26}$

The primary outcome was hospitalization for Covid-19, defined as an inpatient discharge diagnosis with ICD-10-CM code U07.1. A recent study reported that this code had a sensitivity of $98 \%$ and a positive predictive value of $91.5 \% .{ }^{27}$ Secondary outcomes focused on severe complications of Covid-19 hospitalization and included ICU admission, invasive mechanical ventilation, or death during hospitalization or within 7 days of discharge, and a composite of these three (Supplement Table 2). Our analysis found that $83 \%$ of patients who died during the week following Covid-19 hospitalization had been discharged to hospice, a skilled nursing facility, or a nursing home, supporting the classification of these deaths as Covid-19-related. 
Table 1 Demographic and Clinical Characteristics of Hospitalized Covid-19 Cases and Neighborhood-Matched Controls from US Medicare Data. Additional Variables Adjusted for in the Regression Model are Shown in Supplement Table 1

\begin{tabular}{|c|c|c|c|}
\hline Characteristic (\%) & $\begin{array}{l}\text { Cases } \\
(n= \\
\mathbf{3 5}, \mathbf{3 0 0})\end{array}$ & $\begin{array}{l}\text { Controls } \\
(n= \\
228,228)\end{array}$ & $\begin{array}{l}\text { Standardized } \\
\text { mean } \\
\text { difference }\end{array}$ \\
\hline \multicolumn{4}{|l|}{ Demographics } \\
\hline Age, years, mean (SD) & $\begin{array}{l}79.5 \\
(8.3)\end{array}$ & $\begin{array}{l}77.3 \\
(7.9)\end{array}$ & 0.27 \\
\hline Male ... & 42.6 & 37.7 & 0.10 \\
\hline \multicolumn{4}{|l|}{ Race/ethnicity } \\
\hline White & 63.2 & 70.2 & 0.15 \\
\hline Black & 23.2 & 18.2 & 0.12 \\
\hline Hispanic & 6.5 & 3.9 & 0.12 \\
\hline Other & 7.0 & 7.7 & 0.03 \\
\hline Low income subsidy & 57.4 & 40.3 & 0.35 \\
\hline Nursing home resident & 31.7 & 12.9 & 0.46 \\
\hline \multicolumn{4}{|l|}{$\begin{array}{l}\text { Nursing home days in past } \\
\text { year }\end{array}$} \\
\hline 0 & 58.1 & 83.1 & 0.57 \\
\hline $1-30$ & 5.9 & 2.4 & 0.18 \\
\hline $31+$ & 36.0 & 14.5 & 0.51 \\
\hline \multicolumn{4}{|l|}{$\begin{array}{l}\text { General medical } \\
\text { conditions }\end{array}$} \\
\hline Alcohol dependence & 2.6 & 1.9 & 0.04 \\
\hline Asthma & 11.4 & 9.4 & 0.07 \\
\hline COPD & 28.0 & 17.6 & 0.25 \\
\hline Chronic liver disease & 5.7 & 4.6 & 0.05 \\
\hline $\begin{array}{l}\text { Complicated } \\
\text { hypertension }\end{array}$ & 6.3 & 3.1 & 0.15 \\
\hline Dementia & 37.5 & 16.0 & 0.50 \\
\hline Diabetes & 60.1 & 46.1 & 0.28 \\
\hline Diabetic nephropathy & 22.0 & 11.7 & 0.28 \\
\hline Home oxygen use & 9.5 & 3.9 & 0.23 \\
\hline Hospitalized pneumonia & 8.7 & 2.8 & 0.25 \\
\hline Hyperlipidemia & 79.0 & 78.7 & 0.01 \\
\hline Immunocompromised & 11.6 & 4.1 & 0.28 \\
\hline Malignancy & 16.7 & 15.6 & 0.03 \\
\hline Nicotine dependence & 32.5 & 24.8 & 0.17 \\
\hline Obesity & 28.9 & 25.4 & 0.08 \\
\hline Renal artery stenosis & 1.3 & 0.9 & 0.04 \\
\hline \multicolumn{4}{|l|}{ Renal disease } \\
\hline Acute kidney injury & 22.3 & 8.4 & 0.39 \\
\hline Chronic kidney disease* & 42.0 & 25.4 & 0.36 \\
\hline Dialysis & 4.8 & 1.1 & 0.22 \\
\hline $\begin{array}{l}\text { Cardiovascular disease } \\
\text { Acute myocardial }\end{array}$ & 2.2 & 1.0 & 0.10 \\
\hline infarction & & & \\
\hline $\begin{array}{l}\text { Other ischemic heart } \\
\text { disease }\end{array}$ & 44.4 & 32.3 & 0.25 \\
\hline Atrial fibrillation & 26.5 & 17.3 & 0.22 \\
\hline Heart failure (systolic) & 14.1 & 6.8 & 0.24 \\
\hline $\begin{array}{l}\text { Heart failure (diastolic, } \\
\text { other) }\end{array}$ & 35.5 & 18.6 & 0.39 \\
\hline $\begin{array}{l}\text { Peripheral arterial } \\
\text { disease }\end{array}$ & 52.2 & 34.1 & 0.37 \\
\hline Stroke & 1.6 & 0.6 & 0.09 \\
\hline $\begin{array}{l}\text { Other cerebrovascular } \\
\text { disease }\end{array}$ & 28.1 & 15.8 & 0.30 \\
\hline Frailty score, mean (SD) & $\begin{array}{l}0.31 \\
(0.21)\end{array}$ & $\begin{array}{l}0.20 \\
(0.17)\end{array}$ & 0.60 \\
\hline $\begin{array}{l}\text { Charlson score, mean } \\
(\mathrm{SD})^{\dagger}\end{array}$ & $\begin{array}{l}4.55 \\
(2.83)\end{array}$ & $\begin{array}{l}2.96 \\
(2.50)\end{array}$ & 0.59 \\
\hline $\begin{array}{l}\text { Diabetes complications } \\
\text { severity index, mean } \\
(\mathrm{SD})^{\dagger}\end{array}$ & $\begin{array}{l}4.14 \\
(2.48)\end{array}$ & $\begin{array}{l}3.05 \\
(2.31)\end{array}$ & 0.45 \\
\hline \multicolumn{4}{|l|}{ Medication use } \\
\hline Antiarrhythmics & 5.2 & 3.7 & 0.07 \\
\hline Anticoagulants (oral) & 20.7 & 14.5 & 0.16 \\
\hline Antiplatelet agents & 16.5 & 11.5 & 0.14 \\
\hline \multicolumn{4}{|l|}{ Antidiabetic agents } \\
\hline Insulin & 21.5 & 10.6 & 0.30 \\
\hline Metformin & 23.3 & 21.8 & 0.04 \\
\hline Other & 22.6 & 16.7 & 0.15 \\
\hline Digoxin & 2.3 & 1.6 & 0.05 \\
\hline
\end{tabular}

(continued on next page)
Table 1. (continued)

\begin{tabular}{|c|c|c|c|}
\hline Characteristic (\%) & $\begin{array}{l}\text { Cases } \\
(n= \\
\mathbf{3 5 , 3 0 0 )}\end{array}$ & $\begin{array}{l}\text { Controls } \\
(n= \\
228,228)\end{array}$ & $\begin{array}{l}\text { Standardized } \\
\text { mean } \\
\text { difference }\end{array}$ \\
\hline Fibrates & 3.8 & 3.5 & 0.02 \\
\hline Nitrates & 11.1 & 7.2 & 0.14 \\
\hline Oral corticosteroids s & 3.8 & 1.9 & 0.12 \\
\hline \multicolumn{4}{|l|}{ Pulmonary inhalers } \\
\hline Inhaled corticosteroids & 13.4 & 9.5 & 0.12 \\
\hline Long-acting inhalers & 13.9 & 9.9 & 0.12 \\
\hline Short-acting inhalers & 24.7 & 15.4 & 0.23 \\
\hline \multirow{2}{*}{\multicolumn{4}{|c|}{$\begin{array}{l}2^{\text {nd }} \text {-line antihypertensive } \\
\text { drugs }\end{array}$}} \\
\hline & & & \\
\hline Beta blockers & 49.5 & 43.1 & 0.13 \\
\hline Loop diuretics & 27.1 & 15.1 & 0.30 \\
\hline Direct vasodilators & 12.5 & 7.0 & 0.18 \\
\hline $\begin{array}{l}\text { Mineralocorticoid } \\
\text { receptor blockers }\end{array}$ & 4.2 & 3.3 & 0.05 \\
\hline Other & 8.3 & 7.3 & 0.04 \\
\hline
\end{tabular}

$S D$ standard deviation, COPD chronic obstructive pulmonary disease ${ }^{*}$ Chronic kidney disease (CKD) was included in the model as a 3-level categorical variable: no CKD, CKD without proteinuria, and CKD with proteinuria

${ }^{\dagger}$ Charlson score and the adapted diabetes complications severity index were modeled categorically; distributions can be found in Supplement Table 1

$\neq$ Unless otherwise noted, medication use is defined as $\geq 1$ day of use in the 365 days prior to index

$\checkmark$ Defined as $\geq 15$ days of use in the 30 days prior to index

"Defined as $\geq 1$ day of use in the 30 days prior to index

Cases and matched controls were assessed for differences in covariate distributions using standardized mean differences. ${ }^{28}$ The primary analysis was conducted in two stages. In the first stage, multivariable conditional logistic regression was used to examine the association of ACEIs or ARBs with hospitalization for Covid-19. In the second stage, we estimated the risk of ICU admission, invasive mechanical ventilation, or death, as well as a composite of these three. For these analyses, we ran multiple multivariable conditional logistic regression models, with cases assigned their most severe outcome. This was equivalent to fitting a conditional multinomial logistic regression model (Supplement Appendix). ${ }^{29}$ Our findings were reported as odds ratios (ORs) and 95\% confidence intervals (CI).

Because ACEIs and ARBs have guideline-specified indications for use that could lead to unmeasured confounding, a secondary analysis excluded patients with guidelinerecommended indications for ACEI/ARB therapy: systolic heart failure (as a surrogate for heart failure with reduced ejection fraction), diabetes with proteinuria or with moderate to severe chronic kidney disease (CKD), and moderate to severe CKD with proteinuria (regardless of diabetes status). ${ }^{30-32}$ In addition, we excluded patients with renal artery stenosis in whom ACEI/ ARB therapy can either be first-line or contraindicated depending on the severity of disease. Subgroup analyses examined the consistency of results by age, sex, race, nursing home residence, presence of diabetes or cardiovascular disease or chronic obstructive pulmonary disease or $\mathrm{CKD}$, and geographic region. We also investigated for a dose effect by subgrouping to high- and 
low-dose exposure groups. Sensitivity analyses varied the case definition by including cases with ongoing hospitalizations identified through professional services claims; evaluated the impact of claims lag by using a study end date that was 2 weeks earlier; varied the number of days of treatment required to be classified as exposed; restricted the analysis to patients using only one first-line antihypertensive agent (second-line co-treatments were permitted); restricted the mortality outcome to inpatient deaths; and assessed the potential for unmeasured confounding using the $E$-value, ${ }^{33}$ which is the minimum strength of residual confounding required to fully explain away the observed treatment effect. We also conducted post hoc analyses where (1) we used the nursing home-reported mailing address instead of the beneficiary-reported mailing address among beneficiaries who resided in a nursing home on the index date, and (2) we repeated the monotherapy analysis stratified by race.

This project was classified as public health surveillance by FDA and was exempt from review by the agency's institutional review board. Analyses were performed using SAS v 9.4 (SAS Institute Inc., Cary, NC) and R 3.6.0 (R Foundation for Statistical Computing, Vienna, Austria).

\section{RESULTS}

Of 35,348 patients hospitalized with Covid-19, 35,300 $(99.9 \%)$ were successfully matched to 228,228 controls on calendar date and neighborhood of residence (mean $=6.5$ controls per case). Among cases, the median age was 79 years, $57.4 \%$ were female, and the median duration of hospitalization was 8 days (interquartile range 5-12). Compared with controls, case patients were older and more likely to be male, Black or Hispanic, and lower income, and to reside in a nursing home (Table 1). Case patients were also more likely to have a wide variety of medical comorbidities, to have been treated with a variety of chronic medications, and to have had more hospitalizations and emergency department visits in the prior year (Table 1, Supplement Table 1).

Angiotensin-converting enzyme inhibitors and ARBs were associated with a slight but significant reduction in risk of Covid-19 hospitalization compared with other first-line antihypertensive drugs [OR $(95 \% \mathrm{CI})=0.95(0.92-0.98)$ for ACEIs and 0.94 (0.90-0.97) for ARBs] (Fig. 1, Supplement Table 3). Similar results were obtained for the secondary outcomes of ICU admission, invasive mechanical ventilation use, and death. There were no significant differences in risk between ACEIs and ARBs. In a prespecified secondary analysis that excluded patients with specific indications for ACEIs or ARBs, the OR (95\% CI) for Covid-19 hospitalization was $0.93(0.89-0.98)$ for ACEIs and $0.92(0.88-0.96)$ for ARBs.

There was no evidence of increased Covid-19 hospitalization risk with ACEIs or ARBs in subgroups defined by race or nursing home residence, or when stratified by higher or lower dose (Figs. 2 and 3). With age, there was small but statistically significant effect modification for the comparison of ACEIs vs. ARBs. Sensitivity analyses were consistent with the primary analysis (Fig. 4). However, the monotherapy analysis found that $\mathrm{CCBs}$ were associated with a small increased risk of Covid-19 hospitalization compared with ACEIs [OR (95\% CI) 1.09 (1.04-1.14)], ARBs [1.10 (1.05-1.15)], and thiazides [1.11 (1.03-1.19)] (Fig. 5). The post hoc monotherapy analysis stratified by race showed qualitatively similar results without evidence of effect modification by race (Supplement Figure 2). For the primary analysis of Covid-19 hospitalization risk, the $E$-value for ACEIs was 1.29 and for ARBs, 1.33, indicating that a small degree of residual confounding could explain the observed effects.

\section{DISCUSSION}

In our primary analysis, we found that ACEIs and ARBs were associated with a slight reduction in risk of Covid-19 hospitalization compared with other first-line antihypertensive agents and that there was no difference in risk between ACEIs and ARBs. This apparent protective effect was explained by the prespecified monotherapy analysis finding that $\mathrm{CCB}$ use was associated with a small increased risk of Covid-19 hospitalization compared with ACEI, ARB, or thiazide diuretic use. Among these latter first-line agents, the risk of Covid-19 hospitalization was comparable. Risk of ICU admission, invasive mechanical ventilation, and death was similar among first-line antihypertensive agents in our primary analysis. Findings from both the prespecified subgroup and the post hoc monotherapy analysis stratified by race found no evidence of effect differences by race, and the effect on Covid-19 hospitalization risk was similar in subgroups defined by sex, nursing home status, medical comorbidities, and dose of ACEI or ARB. However, there was possible effect modification by age, with a small reduction in risk of Covid-19 hospitalization among ACEIcompared with ARB-treated patients aged 66-74 years, and a reversal of this pattern in patients aged 75 years and older. If this is a real effect, our data indicate that it is very small. The secondary analysis, restricted to patients with hypertension but without other specific indications for ACEIs or ARBs, yielded results indistinguishable from the primary analysis, and suggested that confounding by indication was unlikely. ${ }^{18}$

The finding of a small increase in Covid-19 hospitalization risk with CCBs should be interpreted cautiously as it was not our primary hypothesis and could have arisen from residual confounding. The effect of CCBs on Covid-19 risk was examined in a handful of other published larger observational studies. In several of these, CCBs were not associated with an increased risk of Covid-19 infection or hospitalization, ${ }^{10,13}$ or with all-cause mortality following Covid-19 hospitalization. ${ }^{15}$ However, in one study, CCBs were associated with severe Covid-19 illness compared with non-users in a subgroup analysis restricted to patients with hypertension. ${ }^{7}$ In another, a non-significant increased risk of Covid-19 hospitalization was observed with CCBs compared with ACEIs but not 


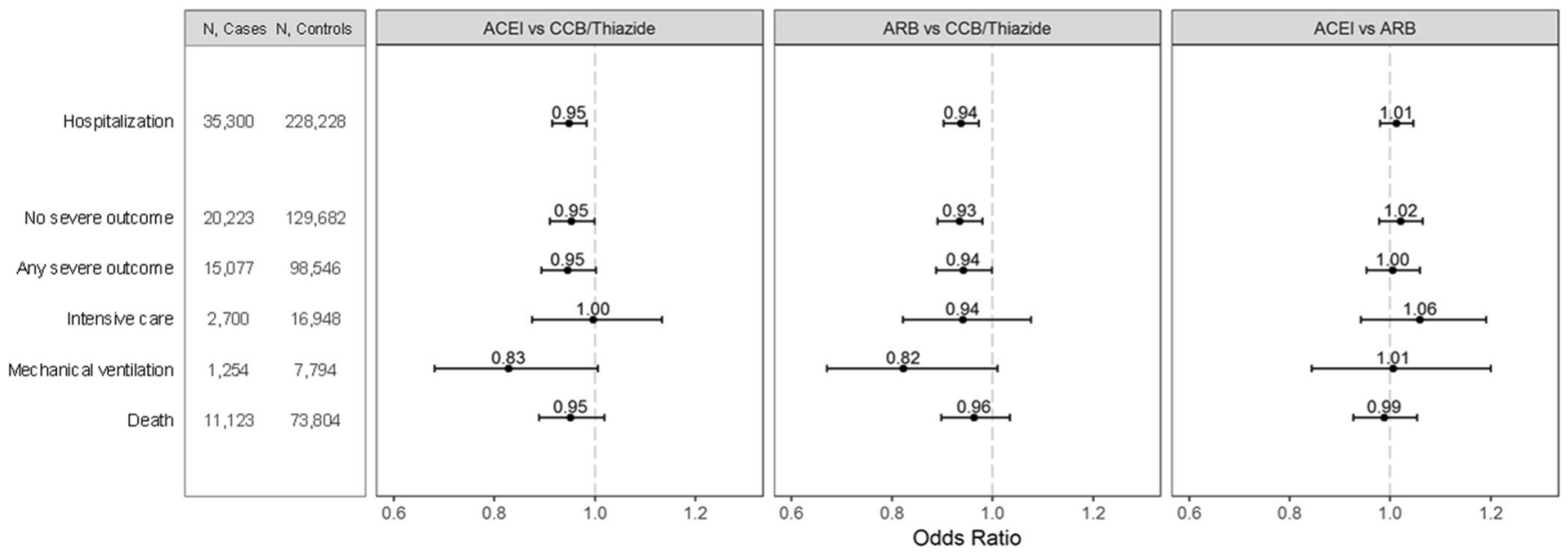

Figure 1 Adjusted odds ratios (95\% confidence intervals) for Covid-19 hospitalization and more serious outcomes in older Medicare beneficiaries treated with a first-line antihypertensive medication.

ARBs. ${ }^{14}$ More recently, a nationwide study from France found increased risks of Covid-19 hospitalization and of a composite outcome of intubation or death with CCBs compared separately with ACEIs and ARBs. ${ }^{17}$ The mechanism by which CCBs might increase Covid-19 risk is unclear.

Our study had many unique strengths that addressed limitations of previous studies evaluating ACEIs, ARBs, and Covid19-related outcomes (Supplement Table 4). It was substantially larger than previously published studies, leading to precise estimates of risk with narrow $95 \%$ confidence intervals. It was national in scope and, by matching on neighborhood and calendar date, was the only study to adjust for the Covid-19 circulation rate, which waxed and waned across the USA as the pandemic evolved. Most of the larger published observational studies had a non-user reference group ${ }^{7-9,11-13}$ and none required treatment with ACEIs or ARBs at the time of Covid-19

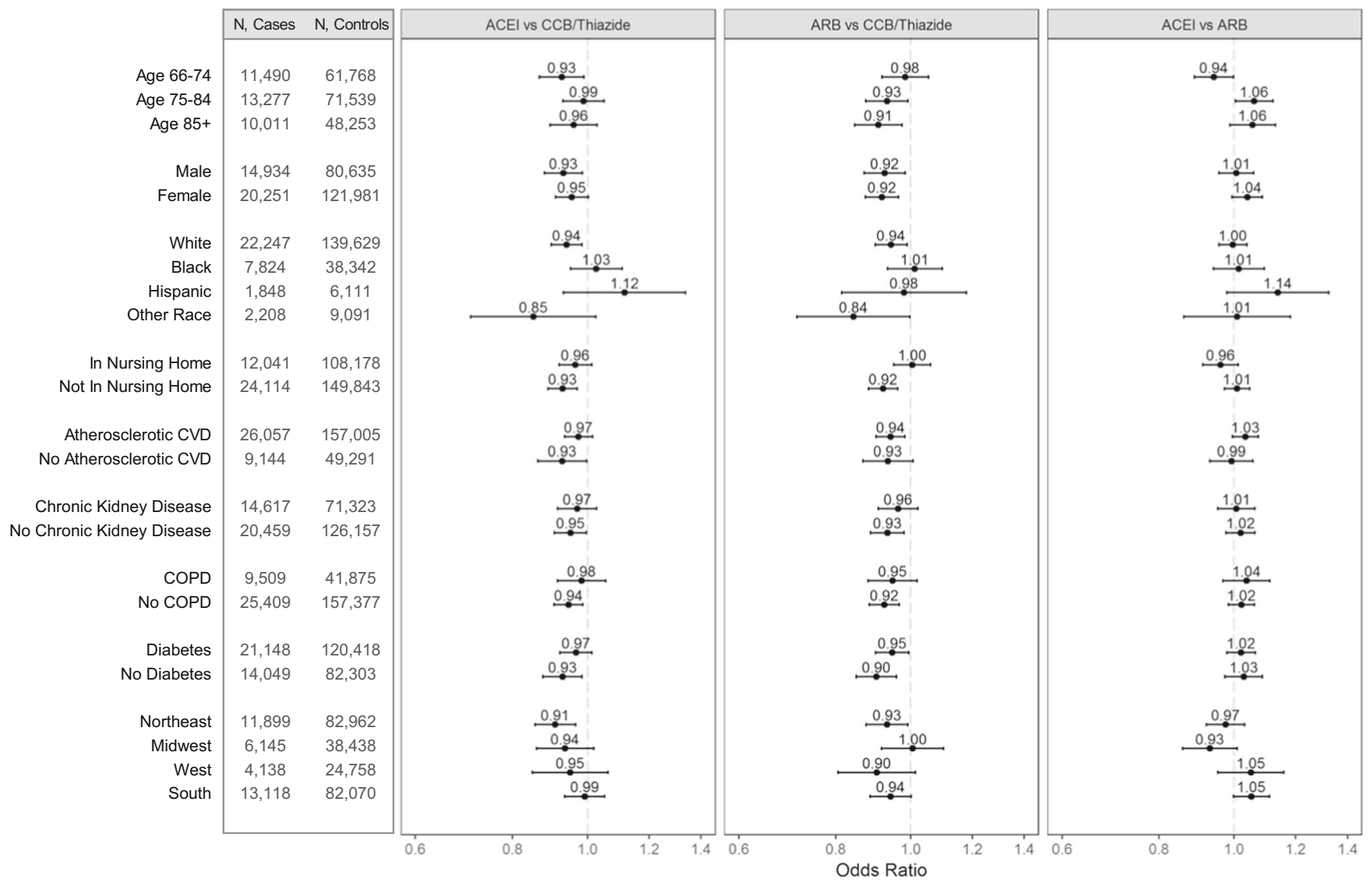

Figure 2 Subgroup analyses showing adjusted odds ratios and 95\% confidence intervals for Covid-19 hospitalization in older Medicare beneficiaries treated with first-line antihypertensive medications. Abbreviations: CVD, cardiovascular disease; COPD, chronic obstructive pulmonary disease. 


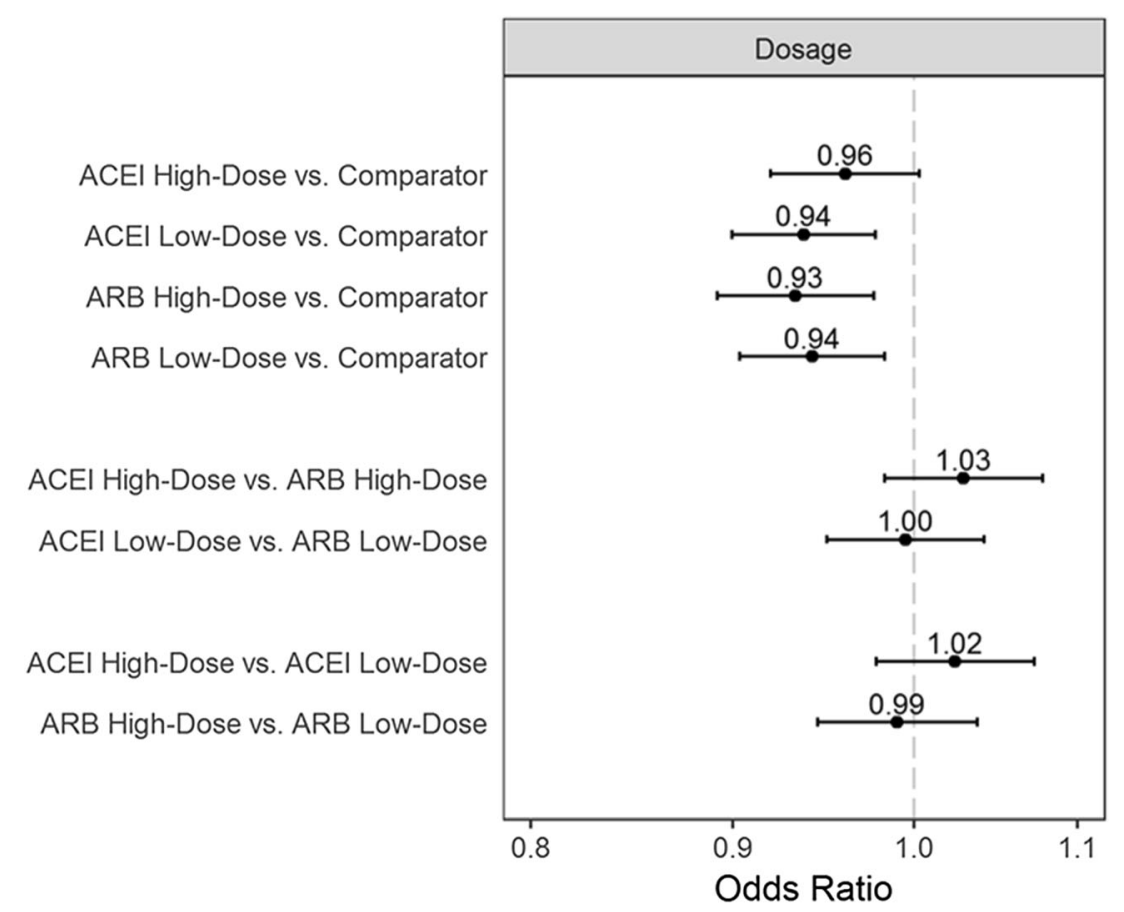

Figure 3 Adjusted odds ratios and 95\% confidence intervals for risk of Covid-19 hospitalization by dose of angiotensin-converting enzyme inhibitor or angiotensin receptor blocker.

diagnosis, ${ }^{7-17}$ increasing the potential for exposure misclassification bias toward the null. Our study required medication supply with a first-line antihypertensive at the time of likely exposure to SARS-CoV-2, thereby reducing the risk of exposure misclassification bias or confounding by indication. ${ }^{18}$ The larger published observational studies were generally limited to a small slice of Covid-19-related outcomes, including a Covid-positive test, ${ }^{7,8,13,14}$ Covid-related hospitalization, ${ }^{13,14,16,17}$ Covid-related death, ${ }^{14,15,16}$ or a mix of severe outcomes. ${ }^{7-11,17}$ None examined the full spectrum of serious Covid-related outcomes. Our study was the only one to examine Covid-related hospitalization as well as more severe outcomes of ICU admission, invasive mechanical ventilation, and death, and had substantial statistical power for each of these outcomes. By assigning cases to their most severe outcome, our analysis recognized that the severity of outcomes is

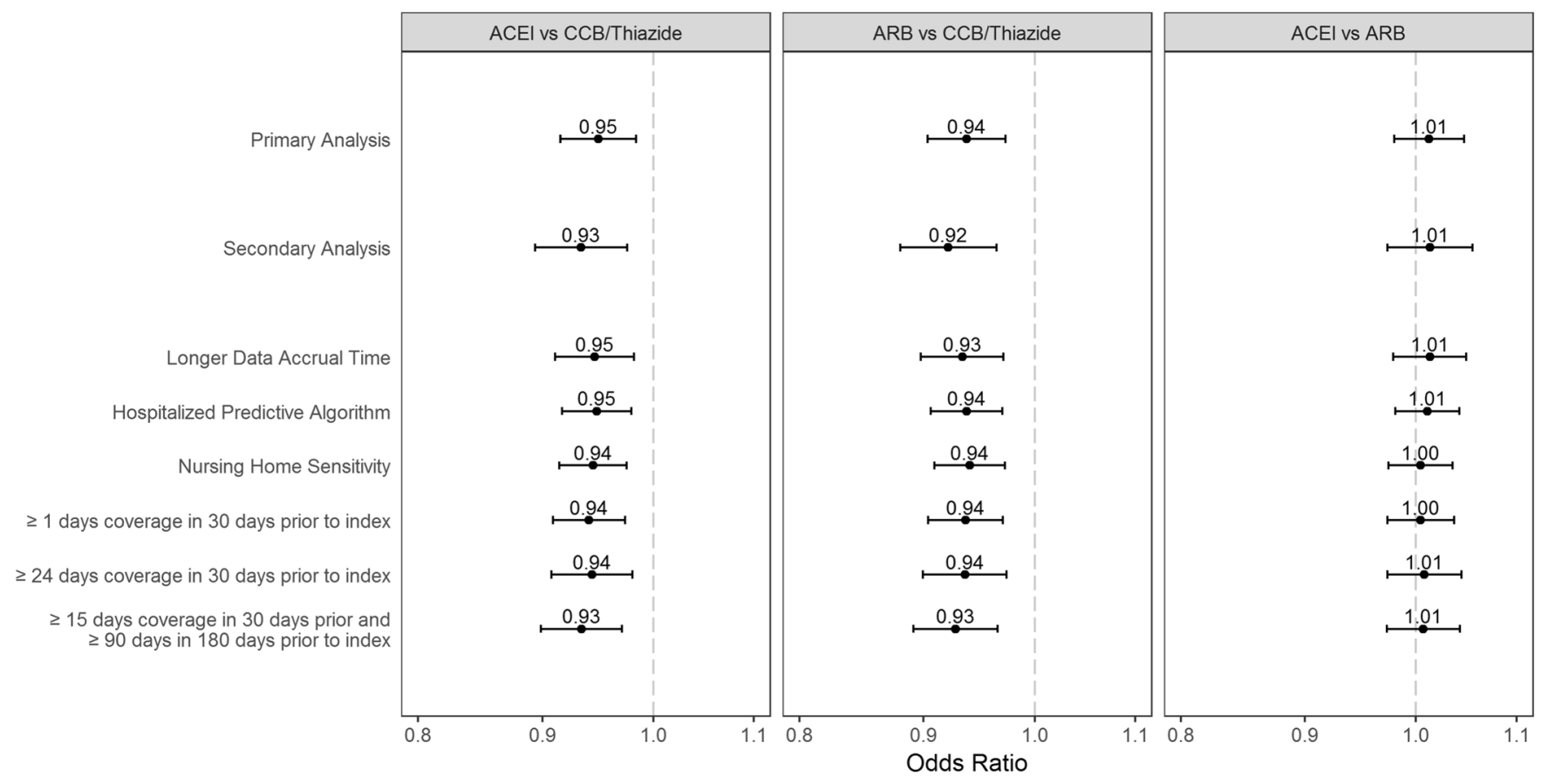

Figure 4 Secondary and sensitivity analysis results showing adjusted odds ratios and $95 \%$ confidence intervals for risk of Covid-19 hospitalization in older Medicare beneficiaries treated with first-line antihypertensives. 


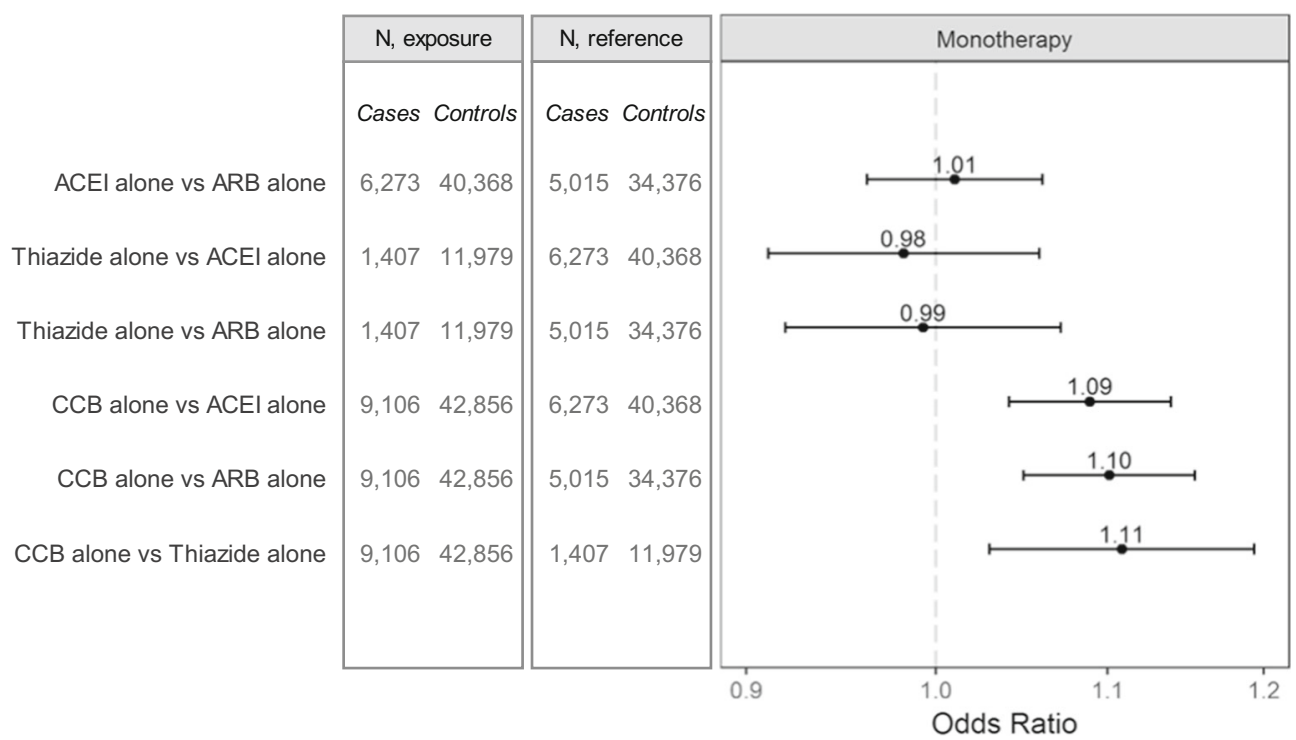

Figure 5 Adjusted odds ratios and 95\% confidence intervals for risk of Covid-19 hospitalization among patients with monotherapy treatment with a first-line antihypertensive medication.

linked for a given individual. Of note, only one other study examined the outcomes of ICU admission and mechanical ventilation individually, reporting a significant increase in ICU admission risk with ACEIs but not ARBs. ${ }^{8}$ This study had insufficient statistical power to exclude a 2- to 2.9-fold increase in risk for these outcomes. Our study provides strong evidence that ACEIs and ARBs are not associated with increased risks of Covid-related ICU admission, invasive mechanical ventilation, or death, or with a composite of these. Only one other larger study, from the UK, explored the effect of race, reporting that ACEIs and ARBs were associated with an increased risk of Covid-19 in Black Africans. ${ }^{11}$ Our study was substantially larger and found no evidence of differential Covid-19 hospitalization risk with ACEIs or ARBs based on race. One previous study examined ACEIs and ARBs combined and found no evidence of a dose effect on risk of Covid19 hospitalization, although this analysis was underpowered and could not exclude a 2.1 -fold increase in risk. ${ }^{13}$ Our study examined ACEIs and ARBs individually and found no evidence of a dose effect with narrow confidence intervals. Finally, our study was sufficiently powered to show that ACEIs and ARBs were not associated with increased Covid-19 hospitalization risk among nursing home residents. No other observational study has examined the effect of ACEIs and ARBs in this vulnerable and high-risk population.

Our study had several limitations. It was not a randomized trial and thus could be subject to residual confounding by unmeasured patient characteristics. We sought to minimize this by restricting the study to patients with hypertension who had a recently filled prescription for a first-line antihypertensive medication. In a secondary analysis, we further restricted to the subset of patients without a specific indication for ACEIs or ARBs. Our study was based on Medicare claims data and thus lacked granular data related to the severity of comorbidities or the quality of blood pressure control. To mitigate this, we adjusted for a wide range of medications used to treat various comorbidities, including non-first-line antihypertensives and total number of antihypertensive classes prescribed, and we also adjusted for health care utilization in the prior year. Although we required all cases and controls to have a sufficient supply of a first-line antihypertensive drug and to have been treated with that medication for $\geq 15$ of the 30 days preceding the index date, we cannot exclude the possibility of non-adherence in some patients. The impact of non-adherence would be to bias any observed associations toward the null. To test for this, we performed a sensitivity analysis that required all cases and controls to have available days' supply of their antihypertensive in both the month before and during the 3 to 6 months prior to the index date, with no change in our results.

In summary, we found that ambulatory treatment of older hypertensive patients with ACEIs or ARBs was not associated with an increased risk of hospitalization for Covid-19 or of hospitalization involving ICU admission, invasive mechanical ventilation, or death. Calcium channel blockers may be associated with a small increase in Covid-19 hospitalization risk compared with ACEIs, ARBs, or thiazide diuretics, but unmeasured confounding cannot be excluded.

Corresponding Author: David J. Graham, MD, MPH; Office of Surveillance and Epidemiology, Center for Drug Evaluation and Research, US Food and Drug Administration, 10903 New Hampshire Ave, Building 22, Room 4314, Silver Spring, MD 20993, USA (e-mail: david.graham1@fda.hhs.gov).

Supplementary Information The online version contains supplementary material available at https://doi.org/10.1007/s11606-02107155-Z.

Funding This study was supported by the US Food and Drug Administration (FDA) through an interagency agreement with the Centers for Medicare \& Medicaid Services (CMS). 


\section{Declarations:}

Conflict of Interest: The authors declare that they do not have a conflict of interest.

Role of the Funder/Sponsor: The authors are employees or contractors of the FDA or the CMS; however, other officials at the FDA and the CMS had no role in the design and conduct of the study; collection, management, analysis, and interpretation of the data; preparation, review, or approval of the manuscript; and decision to submit the manuscript for publication.

Disclaimer: The views expressed are the authors' and not necessarily those of the Centers for Medicare \& Medicaid Services, the Food and Drug Administration, or the Department of Health and Human Services.

\section{REFERENCES}

1. Hoffmann M, Kleine-Weber H, Schroeder S, et al. SARS-CoV-2 cell entry depends on ACE2 and TMPRSS2 and is blocked by a clinically proven protease inhibitor. Cell. 2020;181(2):271-280.e8.

2. Bavishi C, Maddox TM, Messerli FH. Coronavirus disease 2019 (Covid19) infection and the renin angiotensin system blockers. JAMA Cardiol. 2020;5(7):745-747.

3. Tikellis C, Thomas MC. Angiotensin-converting enzyme 2 (ACE2) is a key modulator of the renin angiotensin system in health and disease. Int $J$ Pept. 2012;2012:256-294.

4. Vaduganthan M, Vardeny O, Michel T, McMurray JJV, Pfeffer MA, Solomon SD. Renin-angiotensin-aldosterone system inhibitors in patients with Covid-19. N Engl J Med. 2020;382(17):1653-1659.

5. Hales CM, Servais J, Martin CB, Kohen D. Prescription drug use among adults aged 40-79 in the United States and Canada. NCHS Data Brief. 2019;347:1-8.

6. Mackey K, King VJ, Gurley S, et al. Risks and impact of angiotensinconverting enzyme inhibitors or angiotensin-receptor blockers on SARSCoV-2 infection in adults. A living systematic review. Ann Intern Med. 2020;173: 195-201

7. Reynolds HR, Adhikari S, Pulgarin C, et al. Renin-angiotensinaldosterone system inhibitors and risk of Covid-19. N Engl J Med. 2020;382(25):2441-2448.

8. Mehta N, Kalra A, Nowacki AS, et al. Association of use of angiotensinconverting enzyme inhibitors and angiotensin II receptor blockers with testing positive for coronavirus disease 2019 (COVID-19). JAMA Cardiol. 2020; https://doi.org/10.1001/jamacardio.2020.1855.

9. Jung SY, Choi JC, You SH, Kim WY. Association of renin-angiotensinaldosterone system inhibitors with coronavirus disease 2019 (COVID19)-related outcomes in Korea: a nationwide population-based cohort study. Clin Infect Dis. 2020;71(16):2121-2128.

10. Fosbøl EL, Butt JH, Østergaard L, et al. Association of angiotensinconverting enzyme inhibitor or angiotensin receptor blocker use with COVID-19 diagnosis and mortality. JAMA. 2020;324(2):168-177.

11. Hippisley-Cox J, Young D, Coupland C et al. Risk of severe COVID-19 disease with ACE inhibitors and angiotensin receptor blockers: cohort study including 8.3 million people. Heart. 2020;106(19):1503-1511.

12. Chodick G, Nutman A, Yiekutiel N, Shalev V. Angiotensin-converting enzyme inhibitors and angiotensin-receptor blockers are not associated with increased risk of SARS-CoV-2 infection. J Trav Med. 2020;27(5): 1-3. doi: https://doi.org/10.1093/jtm/taaa069.

13. Dublin S, Walker R, Floyd JS, et al. Renin-angiotensin-aldosterone system inhibitors and COVID-19 infection or hospitalization: a cohort study. Am J Hypertens. 2020; doi: https://doi.org/10.1093/ajh/ hpaa168

14. De Abajo FJ, Rodriguez-Martín S, Lerma V, et al. Use of reninangiotensin-aldosterone system inhibitors and risk of COVID-19 requiring admission to hospital: a case-population study. Lancet. 2020;395:1705-1714.
15. Trifirò G, Massari M, Da Cas R, et al. Renin-angiotensinaldosteronesystem inhibitors and risk of death in patients hospitalized with COVID-19: a retrospective Italian cohort study of 43,000 patients. Drug Safety. 2020:43:1297-1308.

16. Khera R, Clark C, Lu Y, et al. Association of angiotensin-converting enzyme inhibitors and angiotensin receptor blockers with the risk of hospitalization and death in hypertensive patients with Coronavirus disease-19. J Am Heart Assoc. 2021; doi: https://doi.org/10.1161/ JAHA.120.018086.

17. Semenzato L, Botton J, Drouin J, et al. Antihypertensive drugs and COVID-19 risk: a cohort study of 2 million hypertensive patients. Hypertension. 2021;77:833-842.

18. Psaty BM, Koepsell TD, Lin D, et al. Assessment and control for confounding by indication in observational studies. J Am Geriatr Soc. 1999;47:749-754

19. Richardson, D B. An incidence density sampling program for nested casecontrol analyses. Occup Environ Med. 2004;61(12):e59.

20. Segal JB, Chang HY, Du Y, Walston JD, Carlson MC, Varadhan R. Development of a claims-based frailty indicator anchored to a wellestablished frailty phenotype. Med Care. 2017;55(7):716-722.

21. Segal JB, Huang J, Roth DL, Varadhan R. External validation of the claims-based frailty index in the National Health and Aging Trends Study cohort. Am J Epidemiol. 2017;186(6):745-747.

22. Deyo RA, Cherkin DC, Ciol MA. Adapting a clinical comorbidity index for use with ICD-9-CM administrative databases. J Clin Epidemiol. 1992:45(6):613-619.

23. Quan $\mathrm{H}$, Sundararajan $\mathrm{V}$, Halfon $\mathrm{P}$, et al. Coding algorithms for defining comorbidities in ICD-9-CM and ICD-10 administrative data. Med Care. 2005;43(11):1130-1139.

24. Young BA, Lin E, Von Korff M, et al. Diabetes complications severity index and risk of mortality, hospitalization, and healthcare utilization. Am J Manag Care. 2008;14(1):15-23.

25. Chang HY, WeinerJP, Richards TM, Bleigh SN, Segal JB. Validating the adapted diabetes complications severity index in claims data. Am J Manag Care. 2012;18(11):721-726.

26. Mancia G, ReaF, Ludergnani M, Apolone G, Corrao G. Renin-algiotensinaldosterone system blockers and the risk of Covid-19. N Engl J Med. 2020;382(25):2431-2440.

27. Kadri SS, Gundrum J, Warner S, et al. Uptake and accuracy of the diagnosis code for COVID-19 among US hospitalizations. JAMA. 2020;324(24):2553-2554.

28. Austin PC. Balance diagnostics for comparing the distribution of baseline covariates between treatment groups in propensity-score matched samples. Stat Med. 2009:28:3083-3107.

29. Mukherjee B, Liu I, Sinha S. Analysis of matched case-control data with multiple ordered disease states: possible choices and comparisons. Stat Med. 2007;26:3240-3257.

30. KDIGO 2021 Clinical Practice Guideline for the Management of Blood Pressure in Chronic Kidney Disease. Available at: https://kdigo.org/wpcontent/uploads/2016/10/KDIGO-2021-BP-GL.pdf. Accessed February 25, 2021.

31. Yancy CW, Jessup M, Bozkurt B, Butler J, Casey DE Jr, Colvin MM, et al. 2017 ACC/AHA/HFSA Focused Update of the 2013 ACCF/AHA Guideline for the Management of Heart Failure: A Report of the American College of Cardiology/American Heart Association Task Force on Clinical Practice Guidelines and the Heart Failure Society of America. Circulation. 2017:136(6):e137-e161. doi: https://doi.org/10.1161/CIR. 0000000000000509

32. Whelton PK, Carey RM, Aronow WS, Casey DE Jr, Collins KJ, Dennison Himmelfarb C, et al. 2017 ACC/AHA/AAPA/ABC/ACPM/AGS/APhA/ ASH/ASPC/NMA/PCNA Guideline for the Prevention, Detection, Evaluation, and Management of High Blood Pressure in Adults: Executive Summary: A Report of the American College of Cardiology/American Heart Association Task Force on Clinical Practice Guidelines. Circulation. 2018;138(17):e426-e483. doi: https://doi.org/10.1161/CIR 0000000000000597

33. VanderWeele TJ, Ding P. Sensitivity analysis in observational research: introducing the E-value. Ann Intern Med. 2017;167(4):268-274.

Publisher's Note: Springer Nature remains neutral with regard to jurisdictional claims in published maps and institutional affiliations. 